\title{
Aliasing in the Complex Cepstrum of Linear-Phase Signals
}

\section{Bysted, Tommy Kristensen}

Published in:

Information, Communications and Signal Processing, 1997. ICICS., Proceedings of 1997 International Conference on

Link to article, DOI:

10.1109/ICICS.1997.652264

Publication date:

1997

Document Version

Publisher's PDF, also known as Version of record

Link back to DTU Orbit

Citation (APA):

Bysted, T. K. (1997). Aliasing in the Complex Cepstrum of Linear-Phase Signals. In Information,

Communications and Signal Processing, 1997. ICICS., Proceedings of 1997 International Conference on (Vol. 3, pp. 1598-1602). IEEE. https://doi.org/10.1109/ICICS.1997.652264

\section{General rights}

Copyright and moral rights for the publications made accessible in the public portal are retained by the authors and/or other copyright owners and it is a condition of accessing publications that users recognise and abide by the legal requirements associated with these rights.

- Users may download and print one copy of any publication from the public portal for the purpose of private study or research.

- You may not further distribute the material or use it for any profit-making activity or commercial gain

- You may freely distribute the URL identifying the publication in the public portal

If you believe that this document breaches copyright please contact us providing details, and we will remove access to the work immediately and investigate your claim. 


\title{
Aliasing in the complex cepstrum of linear-phase signals
}

\author{
T.K. Bysted \\ Department of Information Technology \\ Technical University of Denmark \\ Building 344 \\ DK-2800 Lyngby \\ Denmark
}

\begin{abstract}
Assuming linear-phase of the associated time signal this paper presents an approximated analytical description of the unavoidable aliasing in practical use of complex cepstrums. The linear-phase assumption covers two major applications of complex cepstrums which are linear- to minimum-phase FIR-filter transformation and minimum-phase estimation from amplitude specifications. The description is made in the cepstrum domain, the Fourier transformed of the complex cepstrum and in the frequency domain. Two examples are given, one for verification of the derived equations and one using the description to reduce aliasing in minimum-phase estimation.
\end{abstract}

\section{Introduction}

In nonlinear digital signal processing cepstrum analysis has been extensively used since the introduction in 1963 . One of the main reasons is the ability to deconvolve signals which can be useful in some situations.

The investigation in this paper is concentrated on the following two applications using the deconvolution property:

- Linear- to minimum-phase FIR-filter transformation [1][2].

- Minimum-phase estimation from amplitude specifications [3][4].

Both applications use the assumption that the associated signal in the time domain is a linear-phase signal and therefore a convolution between a minimum- and a maximum-phase part. In both situations a separation of the two parts is necessary and it is possible to show (see [5]) that a transformation of the linear-phase signal to the complex cepstrum domain automatically performs this separation.

Theoretically this can be done without error. In practice however the involving Fourier transformations are discrete and this introduces aliasing in the complex cepstrum and consequently some kind of error in the final solution (the minimum-phase impulse response or the minimum-phase frequency response). This introduction of aliasing is well-known but nevertheless the error has never been analysed.

In section 2 of this paper approximated analytical equations are derived describing the aliasing in the complex cepstrum domain, the Fourier transformed of the complex cepstrum and the frequency domain. From this it can be seen that the error consists of two parts in the frequency domain. One of these can easily be reduced which is shown in section 3 . In section 4 two examples are given, one for the verification of the derived equations and the other showing the ability to reduce the introduced error in minimum-phase estimation without finding the zeros.

\section{Analytical description of aliasing}

The approximated analytical equations derived in this section assume that the associated time signal is a noncausal linear-phase signal (without zeros on the unit circle). Consequently, the frequency response can be written as [1][5]:

$$
H_{\operatorname{lin}}\left(e^{j \omega}\right)=A \prod_{i=1}^{M_{i}}\left(1-a_{i} e^{-j \omega}\right) \prod_{i=1}^{M_{0}}\left(1-b_{i}^{-1} e^{j \omega}\right)
$$

where $M_{i}=M_{o}$ is the number of zeros inside and outside the unit circle and $a_{i}=\frac{1}{b_{i}^{*}}$. It is assumed that $M_{i}=M_{o}<\frac{N-1}{2}$ and consequently (1) can be transformed to the time domain using an $N$ point discrete Fourier transform (DFT) without losing information.

The theoretical complex cepstrum of (1) is equal [1][5]:

$$
\hat{h}_{\operatorname{lin}}(n)= \begin{cases}-\sum_{i=1}^{M_{i}} \frac{a_{i}^{n}}{n} & n>0 \\ \log A & n=0 \\ \sum_{i=1}^{M_{o}} \frac{b_{i}^{n}}{n} & n<0\end{cases}
$$

which is an infinite sequence decreasing as $\frac{\alpha^{n}}{n}$ where $\alpha=\max \left(\left|a_{i}\right|, \frac{1}{\left|b_{i}^{*}\right|}\right)$.

Computation of the theoretical complex cepstrum requires the Fourier transform, but in practice it is replaced by the DFT. Due to this the obtained cepstrum 
(called $\hat{h}_{p, \operatorname{lin}}(n)$ ) is a time-aliased version of the true cepstrum $\hat{h}_{\text {lin }}(n)$ [5][6][7]:

$$
\hat{h}_{p, \operatorname{lin}}(n)=\sum_{k=-\infty}^{\infty} \hat{h}_{\mathrm{lin}}(n+k N)
$$

Combining (2) and (3) the time-aliased complex cepstrum can be written as:

$$
\hat{h}_{p, \operatorname{lin}}(n)= \begin{cases}-\sum_{i=1}^{M_{i}} \frac{a_{i}^{n}}{n}+\delta_{n>0} & 1 \leq n \leq \frac{N-1}{2} \\ \log A+\delta_{n=0} & n=0 \\ \sum_{i=1}^{M_{o}} \frac{b_{i}^{n}}{n}+\delta_{n<0} & -1 \leq n \leq-\frac{N-1}{2}\end{cases}
$$

where

$$
\begin{aligned}
& \delta_{n>0}=-\sum_{k=1}^{\infty} \sum_{i=1}^{M_{i}} \frac{a_{i}^{n+k N}}{n+k N}+\sum_{k=-\infty}^{-1} \sum_{i=1}^{M_{o}} \frac{b_{i}^{n+k N}}{n+k N} \\
& \delta_{n=0}=-\sum_{k=1}^{\infty} \sum_{i=1}^{M_{i}} \frac{a_{i}^{k N}}{k N}+\sum_{k=-\infty} \sum_{i=1}^{M_{o}} \frac{b_{i}^{k N}}{k N} \\
& \delta_{n<0}=\sum_{k=-\infty}^{-1} \sum_{i=1}^{M_{o}} \frac{b_{i}^{n+k N}}{n+k N}-\sum_{k=1}^{\infty} \sum_{i=1}^{M_{i}} \frac{a_{i}^{n+k N}}{n+k N}
\end{aligned}
$$

is the aliasing.

The rest of this paper is concentrated on description of the minimum-phase part $\left(1 \leq n \leq \frac{N-1}{2}\right)$. Similar expressions can be made for the maximum-phase part.

The total aliasing of the minimum-phase cepstrum described in equation (5) can be divided into contributions from zeros inside and outside the unit circle. From (5) it can be seen that aliasing caused by a single zero inside the unit circle is:

$$
-\sum_{k=1}^{\infty} \frac{a_{i}^{n+k N}}{n+k N}=-a_{i}^{n} \sum_{k=1}^{\infty} \frac{a_{i}^{k N}}{n+k N}
$$

and it can be approximated as:

$$
-a_{i}^{n} \sum_{k=1}^{\infty} \frac{a_{i}^{k N}}{n+k N} \approx-a_{i}^{n} \sum_{k=1}^{\infty} \frac{a_{i}^{k N}}{k(n+N)}
$$

resulting in the equation:

$$
-\sum_{k=1}^{\infty} \frac{a_{i}^{n+k N}}{n+k N} \approx \frac{a_{i}^{n}}{\left(1+\frac{n}{N}\right)} \frac{1}{N} \ln \left(1-a_{i}^{N}\right)
$$

Using a similar approximation for a zero outside the unit circle the total aliased minimum-phase part of the cepstrum can be expressed as:

$$
\hat{h}_{p}(n) \approx-\sum_{i=1}^{M_{i}}\left(\frac{a_{i}^{n}}{n}+\frac{a_{i}^{n} K_{a_{i}}}{1+\frac{n}{N}}+\frac{b_{i}^{n} K_{b_{i}}}{1-\frac{n}{N}}\right)
$$

where $1 \leq n \leq \frac{N-1}{2}$ and

$$
\begin{aligned}
K_{a_{i}} & =-\frac{1}{N} \ln \left(1-a_{i}^{N}\right) \quad \text { and } \\
K_{b_{i}} & =-\frac{1}{N} \ln \left(1-b_{i}^{-N}\right)
\end{aligned}
$$

Equation (11) can be transformed to the frequency domain using a DFT followed by the exponential function. First the DFT gives $\hat{H}\left(e^{j \omega_{k}}\right)\left(\omega_{k}=\frac{2 \pi}{N} k\right)$ (called the Fourier transformed of the complex cepstrum) which is approximately equal to:

$$
\begin{aligned}
\hat{H}_{p}\left(e^{j \omega_{k}}\right) \approx \sum_{i=1}^{M_{i}}\left(\ln \left(1-a_{i} e^{-j \omega_{k}}\right)+\right. \\
\quad \sum_{n=\frac{N-1}{2}+1}^{\infty} \frac{a_{i}^{n} e^{-j \omega_{k} n}}{n} \\
-\frac{K_{a_{i}} a_{i} e^{-j \omega_{k}}-\frac{2}{3} K_{a_{i}}\left(a_{i} e^{-j \omega_{k}}\right)^{\frac{N-1}{2}+1}}{1-a_{i} e^{-j \omega_{k}}} \\
\left.-\frac{K_{b_{i}} b_{i} e^{-j \omega_{k}}-2 K_{b_{i}}\left(b_{i} e^{-j \omega}\right)^{\frac{N-1}{2}+1}}{1-b_{i} e^{-j \omega_{k}}}\right)
\end{aligned}
$$

The exponential function will now transform from $\hat{H}_{p}\left(e^{j \omega_{k}}\right)$ to the frequency domain:

$$
H_{p}\left(e^{j \omega_{k}}\right) \approx \prod_{i=1}^{M_{i}}\left(\left(\mathbb{1}-a_{i} e^{-j \omega_{k}}\right) \cdot \Delta_{1} \cdot \Delta_{2}\right)
$$

where

$$
\begin{aligned}
\Delta_{1}= & \frac{1-a_{i}\left(1+K_{a_{i}}\right) e^{-j \omega_{k}}}{1-a_{i} e^{-j \omega_{k}}} \\
& \frac{1-b_{i}\left(1+K_{b_{i}}\right) e^{-j \omega_{k}}}{1-b_{i} e^{-j \omega_{k}}}
\end{aligned}
$$

and

$$
\begin{aligned}
\Delta_{2}= & \left(\prod_{n=\frac{N-1}{2}+1}^{\infty}\left(1+\frac{a_{i}^{n} e^{-j \omega_{k} n}}{n}\right)\right) \\
& \cdot \frac{1-a_{i} e^{-j \omega_{k}}+\frac{2}{3} K_{a_{i}}\left(a_{i} e^{-j \omega_{k}}\right)^{\frac{N-1}{2}+1}}{1-a_{i} e^{-j \omega_{k}}} \\
& \cdot \frac{1-b_{i} e^{-j \omega_{k}}+2 K_{b_{i}}\left(b_{i} e^{-j \omega_{k}}\right)^{\frac{N-1}{2}+1}}{1-b_{i} e^{-j \omega_{k}}}
\end{aligned}
$$

In the above transformation the exponential function has been approximated as $e^{x} \approx 1+x$ in the last three terms of (14). This can be done because $K_{a_{i}} \wedge K_{b_{i}} \ll 1$.

Despite the use of approximations in the derivation of equation (15) the difference between the error described by this model $\left(\Delta_{1}\right.$ and $\left.\Delta_{2}\right)$ and the true error introduced by aliasing is very small. This is verified in Example 1 in section 4 .

Equation (15) shows that the minimum-phase frequency response found from the aliased cepstrum can be divided into three different parts. The first one is the true frequency response while the second and third parts (called $\Delta_{1}$ and $\Delta_{2}$ ) are the introduced error.

There are three reasons for this dividing of the total error into two parts:

1. $\Delta_{1}$ and $\Delta_{2}$ are generated at two different stages. 
2. The two parts introduce two different kinds of error.

3 . One of them can be reduced without any information about the exact location of the zeros. This is shown in section 3 .

The statement in point 1 can be verified from a discrete Fourier transform of equation (11). If this transformation is performed it can be seen that $\Delta_{1}$ is the direct effect of the time-aliasing (see (3)) while $\Delta_{2}$ is due to the limited length $(N)$ of the transformation from $\hat{h}_{p}(n)$ to $\hat{H}_{p}\left(e^{j \omega_{k}}\right)$.

From equation (16) it can be seen that $\Delta_{1}$ is controlled by the constants $K_{a_{i}}$ and $K_{b_{i}}$. In practical situations these two are small and consequently $\Delta_{1}$ will only generate a minor error in the vicinity of the actual zero.

The total contribution from the three terms in $\Delta_{2}$ (see equation (17)) is an oscillating error having a constant amplitude except in the vicinity of the zero where a peak is present. Due to this disturbance of the whole spectrum a reduction of $\Delta_{2}$ is desirable. The chance of reducing this error is investigated in the next section.

The above two statements are visualised in Example 2 in section 4.

\section{Reducing aliasing}

In this section the possibility of reducing aliasing is investigated. From the total frequency domain description in section 2 this seems impossible without finding the zeros.

If the investigation is moved into the time domain and the dividing in the two contributions is retained this section will show:

- Reduction of the $\Delta_{1}$ error requires the zeros of $h_{\operatorname{lin}}(n)$.

- $\Delta_{2}$ can be reduced in the time domain by a truncation.

The assumption $M_{i}=M_{o}<\frac{N-1}{2}$ made in section 2 cause a limitation of the true minimum-phase impulse response to the area $n \in\left[0, \frac{N-1}{2}[\right.$.

From equation (16) it can be seen that the disturbance caused by $\Delta_{1}$ is in the same interval. As a consequence it is not possible to remove $\Delta_{1}$ without finding the zeros.

The disturbance caused by $\Delta_{2}$ is not limited to the above interval. Actually equation (17) shows that the main components will occur outside this area. Truncating the impulse response calculated from $H_{p}\left(e^{j \omega_{k}}\right)$ to:

$$
h_{p, \min }(n)= \begin{cases}h_{p}(n) & n \in\left[0, \frac{N-1}{2}[\right. \\ 0 & n \in\left[\frac{N-1}{2}, N-1\right]\end{cases}
$$

will therefore reduce the error introduced by $\Delta_{2}$.

The possibility of error reduction in the two applications mentioned in the introduction (using the above method) is now investigated. This require discrimination between the two applications.

\begin{tabular}{|c|c|}
\hline$z_{a_{1}}=z_{a_{2}}^{*}=\frac{1}{z_{b_{1}}^{*}}=\frac{1}{z_{b_{2}}}$ & $0.99 \cdot e^{j 2.0780}$ \\
\hline$z_{a_{3}}=z_{a_{4}}^{*}=\frac{1}{z_{b_{3}}^{*}}=\frac{1}{z_{b_{4}}}$ & $0.98 \cdot e^{j 0.8935}$ \\
\hline$z_{a_{5}}=z_{a_{6}}^{*}=\frac{1}{z_{b_{5}}^{*}}=\frac{1}{z_{b_{6}}}$ & $0.97 \cdot e^{j 0.2035}$ \\
\hline$z_{a_{7}}=z_{a_{8}}^{*}=\frac{1}{z_{b_{7}}^{*}}=\frac{1}{z_{b_{8}}}$ & $0.96 \cdot e^{j 1.8309}$ \\
\hline
\end{tabular}

Table 1: Zeros of a linear-phase FIR-filter.

In linear- to minimum-phase FIR-filter transformation the final solution is the minimum-phase impulse response. From this minimum-phase impulse response only the first $M_{i}+1$ points are used. Due to this the truncation described in equation (18) is automatically performed and an additional reduction requires the zeros of $h_{\text {lin }}(n)$.

The desired signal in minimum-phase estimation is $J_{\mathcal{M}}\left(\hat{H}_{p}\left(e^{j \omega_{k}}\right)\right)$. This estimated phase is corrupted by both types of error and consequently a reduction of the error is possible. This is verified in Example 2 where a minimum-phase function is estimated and the introduced aliasing reduced using the above procedure.

The cost of this error reduction is $2\left(N+N \log _{2} N\right)$ flops where one half are used for the transformation to the time domain and the other half used for transformation back to the frequency domain and phase calculation.

\section{Examples}

Two different examples are given in this section.

The first one has two different purposes:

1. to validate equation (15) the analytical description in the frequency domain.

2. to visualize the error introduced by $\Delta_{1}$ and $\Delta_{2}$.

In Example 2 a minimum-phase function is estimated from an amplitude and the introduced error is reduced using the method described in section 3 .

Example 1 For verification of equation (15) a linearphase FIR-filter is generated having the zeros shown in table 1.

From the impulse response the complex cepstrum has been found using $N=512$ points FFT's. The minimumphase part of the complex cepstrum is transformed back to the frequency domain and called $H_{\text {cep }}\left(e^{j \omega_{k}}\right)$. The difference between this true aliased frequency response and the theoretical,one calculated using equation (15) (called $\left.H_{p}\left(e^{j \omega_{k}}\right)\right)$ is the model error. The model error for this example is plotted in figure 1. For comparison the error introduced by aliasing is depicted as well (the difference between $H_{\text {cep }}\left(e^{j \omega_{k}}\right)$ and the true minimumphase amplitude response $\left.H\left(e^{j \omega_{k}}\right)\right)$

The two plots in figure 1 clearly demonstrate that the model error is negligible compared to the error introduced by aliasing. Consequently, the derived equations 

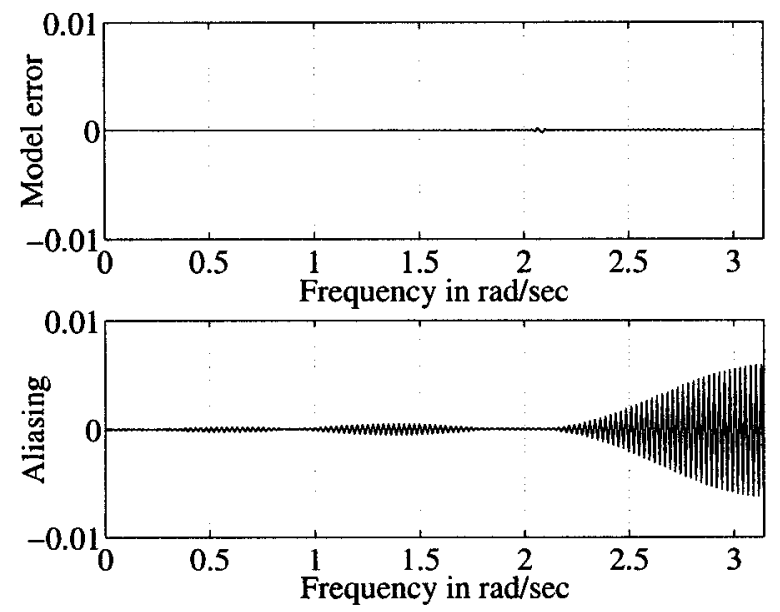

Figure 1: Top: Model error.

Bottom: Error introduced by aliasing.

can be used as an analytical description of aliasing in the complex cepstrum domain (11), in the domain of the Fourier transformed of the complex cepstrum (14) and in the frequency domain (15).

The second part of this example illustrates the error introduced by $\Delta_{1}$ and $\Delta_{2}$ which was described at the end of section 2. $\Delta_{1}$ and $\Delta_{2}$ are calculated from equation (16) and (17) using the zeros shown in table 1. The amplitude and angle of the two errors are plotted in figures 2 and 3.
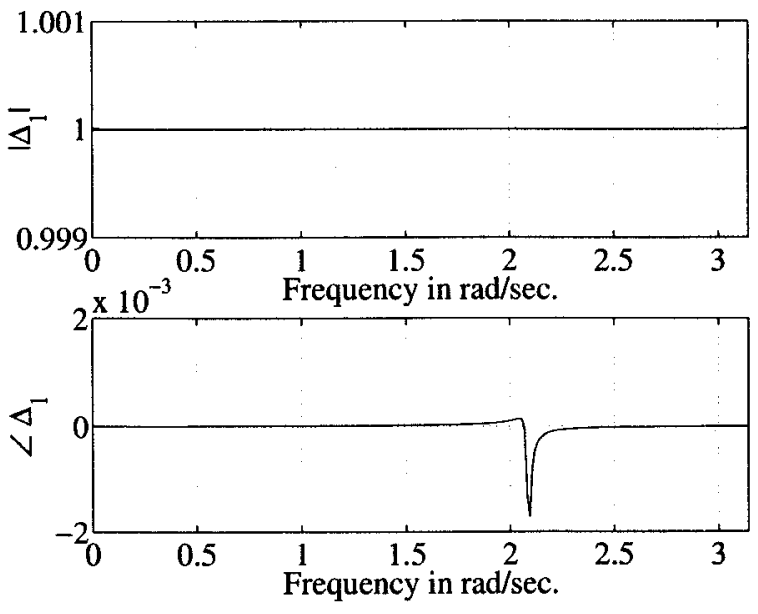

Figure 2: Absolute value and phase of $\Delta_{1}$.

The two figures confirm the two statements from section 2:

- $\Delta_{1}$ error is concentrated in the vicinity of the zeros.

- $\Delta_{2}$ generates an oscillating error having a peak near the zeros but not limited to this area.

As expected the two figures illustrate that the error introduced by the zeros having least distance to the unit
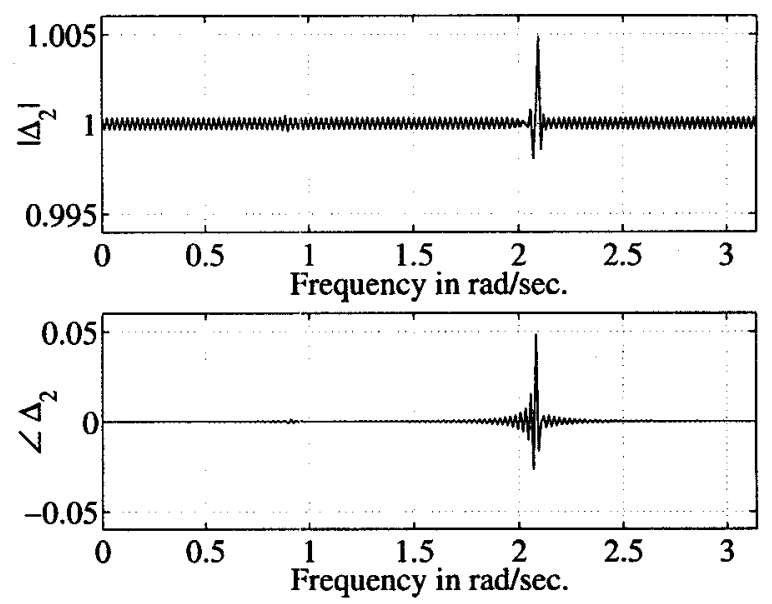

Figure 3: Absolute value and phase of $\Delta_{2}$.

\begin{tabular}{|l|l|}
\hline Length & $20 \mathrm{~km}$ \\
\hline Resistance & $11.2 \frac{\Omega}{\mathrm{km}}$ \\
\hline Capacitance & $50 \frac{\mathrm{nF}}{\mathrm{km}}$ \\
\hline Inductance & $800 \frac{\mu \mathrm{H}}{\mathrm{km}}$ \\
\hline$R_{s}$ (source resistance) & $600 \Omega$ \\
\hline$R_{l}$ (load resistance) & $600 \Omega$ \\
\hline
\end{tabular}

Table 2: Cable data

circle completely dominates the total error. This can be explained by the fact that the complex cepstrum decreases as $\frac{\alpha^{n}}{n}$ where $\alpha=\max \left(\left|a_{i}\right|, \frac{1}{\left|b_{i}^{*}\right|}\right)$.

Example 2 As explained in the beginning of this section the possibility of error reduction in minimumphase estimation is illustrated in this example.

The amplitude used for the estimation is the amplitude of a minimum-phase (FIR) cable-equalizer (opti$\mathrm{mal}$ in the LS-sense). This equalizer is generated from the cable data shown in table $2\left(f_{s}=48 \mathrm{kHz}\right)$ using the method described by Hermann et al [8], which can be summarized as:

1. Design a linear-phase FIR-equalizer $H_{\text {lin }}\left(e^{j \omega}\right)$ having an amplitude $\left|H\left(e^{j \omega}\right)\right|^{2}$ equal to the square of the desired amplitude.

2. Generate a new FIR-equalizer from the zeros of $H_{\text {lin }}\left(e^{j \omega}\right)$ inside the unit circle. This new equalizer $\left(H\left(e^{j \omega_{k}}\right)\right)$ is a minimum-phase FIR-equalizer having the amplitude $\left|H\left(e^{j \omega_{k}}\right)\right|$.

In figure 4 the amplitude of the cable and the amplitude and phase of the designed minimum-phase equalizer are plotted.

The theory of complex cepstrums is used to estimate a minimum-phase function $\left(\mathcal{J}_{\mathfrak{K}}\left(\hat{H}_{p}\left(e^{j \omega_{k}}\right)\right)\right)$ from the amplitude of the cable-equalizer [3][4]. The length of the 

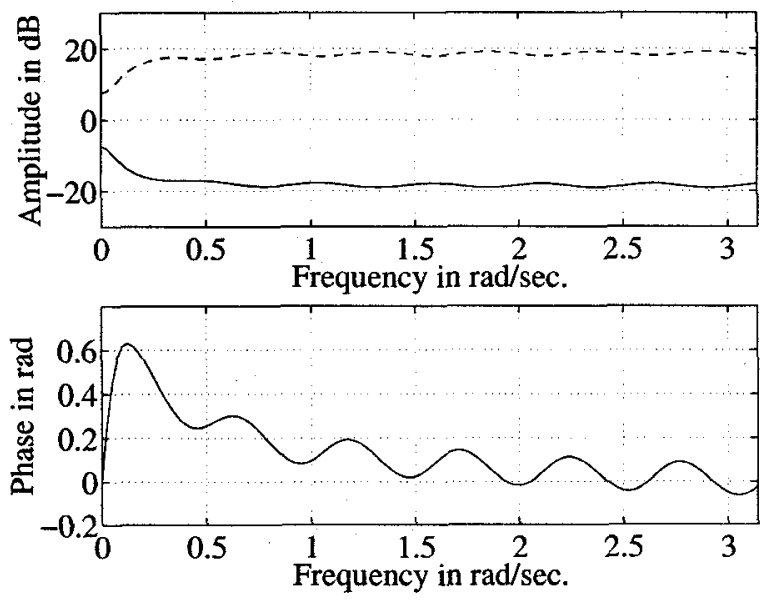

Figure 4: Top: Amplitude response of cable (solid line) and equalizer (dotted line).

Bottom: Phase response of equalizer.

involving discrete Fourier transformations is $N=256$ points.

The error in the estimated phase can be visualised by plotting the difference between the phase of the minimum-phase equalizer $\angle H\left(^{j \omega_{k}}\right)$ and $\mathcal{J}_{\mathcal{M}}\left(\hat{H}_{p}\left(e^{j \omega_{k}}\right)\right)$ which is done in figure 5 . In the same figure the phase error after reduction is depicted.
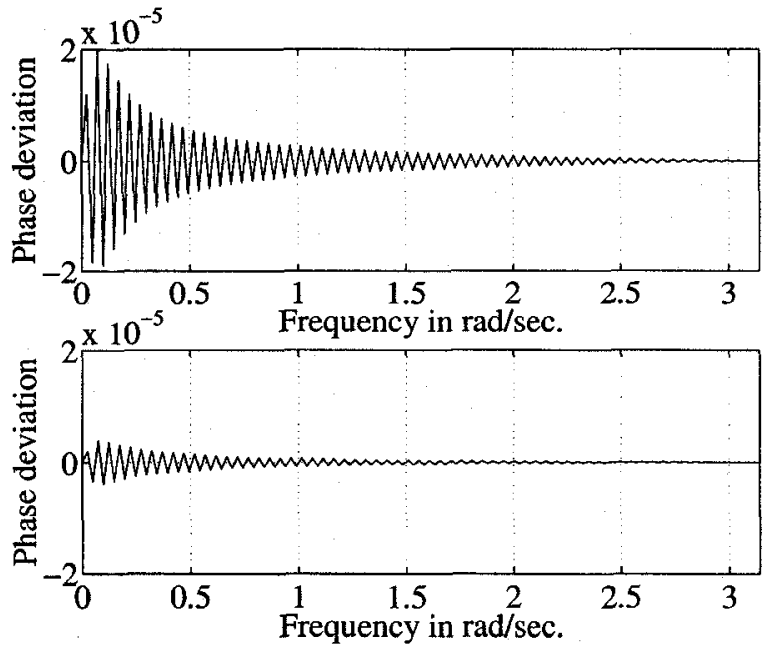

Figure 5: Top: Phase deviation before reduction.

Bottom: Phase deviation after reduction.

As stated the error introduced by aliasing can be reduced by the truncation in the time domain. In this example the reduction is a factor 5 which is obtained using only 4608 flops.

\section{Conclusion}

Approximated analytical equations describing the unavoiable aliasing in practical use of the complex cepstrum are derived assuming that the associated time sig- nal is a linear-phase signal. The description covers the complex cepstrum domain, the domain of the Fourier transformed of the complex cepstrum and the frequency domain.

In the frequency domain the introduced error consists of two parts called $\Delta_{1}$ and $\Delta_{2}$. The error introduced by $\Delta_{1}$ is limited to the vicinity of the zeros while the oscillating error from $\Delta_{2}$ disturbs the whole spectrum.

A total removal of the error requires the exact zeros of the impulse response but it is shown that the oscillating error can be reduced without finding the zeros. The computational cost for this reduction is only $2 \cdot N+2 \cdot N \log _{2} N$ flops where $N$ is the length of the discrete Fourier transformations.

Two different examples are given for verification. The first one verifies the derived equations describing the introduced error and the second one demonstrates the ability to reduce the oscillating error without finding any zeros.

\section{References}

[1] Bysted, T.K., Jensen, K.J. and Gaunholt, H. (1996): Design of Linear- and Minimum-phase FIRequalizers, Proceedings BEC-96,401-404.

[2] Reddy, G.R. (1986): Design of Minimum-phase FIR-Digital Filter Through Cepstrum, Electron. Lett., 22:(1225:1227).

[3] Bysted, T.K. and Gaunholt, H. (1997): MinimumPhase FIR-Equalizer Design Using Phase Estimation, Proceedings ECCTD-97.

[4] Yegnanarayana, B. and Dhayalan, A. (1983): Noniterative Techniques for Minimum Phase Signal Reconstruction From Phase or Magnitude, Proceedings ISCASSP, 639:642.

[5] Oppenheim, A.V. and Schafer, R.W. (1989): Discrete-time signal processing, Prentice Hall.

[6] Childers, D.G., Skinner, D.P. and Kemerait, R.C. (1977): The Cepstrum: A guide to processing, Proc. IEEE, 65:1428-1443.

[7] Pitas, I. and Venetsanopoulos, A.N. (1995): Nonlinear Digital Filters: Principles and Applications, Kluwer Academic Publishers.

[8] Hermann, O. and Schuessler, W. (1970): Design of nonrecursive digital filters with minimum phase, Electron. Lett., 6:329-330. 